\title{
Germanica
}

\section{“Aus nächster Ferne". Josef Winklers Italien-Blicke in Friedhof der bitteren Orangen}

"De loin - un lointain si proche». La perspective italienne de Josef Winkler dans Friedhof der bitteren Orangen

"From far - such a close distance". Josef Winkler's Italian perspective in Friedhof der bitteren Orangen (Graveyard of Bitter Oranges)

\section{Manfred Müller}

\section{OpenEdition}

\section{Journals}

Édition électronique

URL : http://journals.openedition.org/germanica/257

DOI : 10.4000/germanica.257

ISSN : 2107-0784

\section{Éditeur}

Université de Lille

Édition imprimée

Date de publication : 1 juin 2007

Pagination : 59-74

ISBN : 2-913857-19-1

ISSN : 0984-2632

\section{Référence électronique}

Manfred Müller, «"Aus nächster Ferne". Josef Winklers Italien-Blicke in Friedhof der bitteren Orangen », Germanica [Online], 40 | 2007, Online erschienen am: 16 Februar 2010, abgerufen am 06 Oktober 2020. URL : http://journals.openedition.org/germanica/257 ; DOI : https://doi.org/10.4000/germanica.257

Ce document a été généré automatiquement le 6 octobre 2020.

(ㄷ) Tous droits réservés 


\section{"Aus nächster Ferne"1. Josef Winklers Italien-Blicke in Friedhof der bitteren Orangen}

«De loin - un lointain si proche ». La perspective italienne de Josef Winkler dans Friedhof der bitteren Orangen

"From far - such a close distance". Josef Winkler's Italian perspective in Friedhof der bitteren Orangen (Graveyard of Bitter Oranges)

\section{Manfred Müller}

\section{1}

Als Josef Winkler 1990 Friedhof der bitteren Orangen ${ }^{2}$, sein bis dahin sechstes Buch, veröffentlichte, stellten die meisten Rezensenten eilig fest, das Buch füge sich in die "thematische Einheitlichkeit" ${ }^{3}$ der Romane Winklers, eine Einheitlichkeit, die es ermögliche, das Gesamtwerk des Autors als ein einziges, dickes Buch zu lesen ${ }^{4}$. Eine Entwicklung wurde allerdings konstatiert: Bereits im Roman Der Leibeigene habe der Erzähler die "Grenzen seiner inneren Wirklichkeit überschritten", in Friedhof der bitteren Orangen gehe er noch weiter "aus sich heraus":

Der Friedhof der bitteren Orangen verliert sich nicht in hermetischen Traumwelten,

sondern ist ein Journal, ein Tagebuch, das häufig wenigstens an den realen

Alptraum rührt ${ }^{6}$.

Es gehe nun nicht mehr nur um die Kindheit und den Leidensweg des Erzählers, sondern auch um andere, ebenso Schwache.

Als zweite, noch auffälligere "Neuerung" wurde der Wechsel des Schauplatzes bewertet: Zwar berichtet die Erzählerfigur auch in diesem Buch häufig von Kamering, dem Ort ihrer Kindheit, sie selbst befindet sich aber in Italien, dem Schauplatz des weitaus größten Teils des Romans. 
Dass Winkler sich gerade die italienische Hauptstadt ausgesucht hat, ist natürlich kein Zufall: Das "Imperium, [das] Zentrum der katholischen Kirche"' steht wie kaum eine andere Stadt für Themen, die Winklers Literatur bis zu diesem Zeitpunkt bestimmen:

Und während ich den Leibeigenen [...] geschrieben habe [...] [,] da bin ich dann gleichzeitig schon immer wieder nach Venedig und weiter nach Rom hinuntergefahren und da hab ich ja schon parallel das Material für meinen Friedhof der bitteren Orangen gesammelt. Da hab ich ja schon ausprobiert, wovon ich jetzt rede, nämlich das Suchen nach anderen Feldern, nach anderen Themen, die aber selbstverständlich mit den meinen in einem sehr engen Zusammenhang stehen müssen. Mich hat ja dann in Rom der Katholizismus interessiert, ich bin sehr viel im Vatikan gewesen und auf Friedhöfen. Und auf dem großen Markt, auf dem Vittorio Emmanuele, und bin diese Fleisch- und Fischstände entlangspaziert und hab dort das Notizbuch aufgeschlagen und direkt dort geschrieben. [...] Und ich bin halt auch durch unzählige Kirchen gegangen. Also meinen Themenkreis hab ich ja nicht weiß Gott wie erweitert. Das waren ja schon die dörflichen Themen. [...] Nun hab ich das auf das Großstädtische ausgedehnt ${ }^{8}$.

Diese Interviewaussage stellt die Schauplatzwechsel in den Büchern Josef Winklers in einen inhaltlich determinierten Zusammenhang. Seine Figuren verlassen ihren Spielraum nicht, um Neues kennen zu lernen, sondern um bereits zuvor Thematisiertes, jedoch noch nicht Abgeschlossenes aus neuer Perspektive zu erleben und festzuschreiben.

Reisen in das nur wenige Zugstunden entfernte Venedig oder nach Rom sind für den jungen Autor ebenso bedeutsam wie für seine Figuren, sie lassen das südliche Nachbarland allerdings nie als ein herkömmliches Reiseziel erscheinen, sondern als einen Fluchtpunkt, eine Möglichkeit, der Strenge des Ackermanns und der Enge der dörflichen Umgebung zu entkommen. ${ }^{9}$ Zur Begründung seiner Fluchten bemüht Winkler, wie häufig in seinen Texten, ein literarisches Vorbild: "Wer seine Lage nicht zerstört, den zerstört die Lage, so Friedrich Hebbel. Ich zerstörte meine Kärntner Lage und flüchtete nach Rom, und es ist die Frage, wann ich meine römische Lage werde zerstören müssen." (F, S. 315)

In Winklers fünftem Buch, dem Roman Der Leibeigene, wird ein anderer Zusammenhang hergestellt:

Ich werde wieder nach Rom flüchten. Seit Monaten schmachte ich in der Dorfenge dahin, ohne die Haut eines Menschen berührt zu haben. Mit einem Knabenskelett in meinem Katzenmaul werde ich in Rom die Via Regina Margherita entlanglaufen ${ }^{10}$.

Als Flucht aus der Dorfenge ist die Reise offenbar intendiert, schon das erwähnte "Knabenskelett" verweist allerdings darauf, dass vieles mitgeführt wird, was auch das Leben und Schreiben in Kärnten bestimmt hat. Die Themen, die die Trilogie Im wilden Kärnten ${ }^{11}$ bestimmt haben, bleiben im Ausland erhalten: der Tod, die Kirche, die Homosexualität und der Selbstmord zweier Jugendfreunde des Erzählers "Enznsepp", wie er sich selbst, die in Österreichs bäuerlichen Bereichen nach wie vor gebräuchliche, den Hofnamen einbeziehende, Namensgebung verwendend, nennt ${ }^{12}$.

Und so verwundert es nicht, dass er vieles, was ihm in Italien begegnet, auch und vor allem auf Kärnten oder, genauer, auf sich selbst in Kärnten, bezieht:

[I]ch hab auch kein Buch über Rom geschrieben, sondern über mich in Rom. [...] Und später dann bin ich nach Indien gegangen und habe auch kein Buch über Indien geschrieben, sondern ein Buch über ein Thema, das man von mehreren meiner Bücher kennt - über den Tod [... $]^{13}$. 
Beispiele, die diese Interviewaussage untermauern, finden sich in Friedhof der bitteren Orangen zuhauf: Die "Madonna sulla Seggiola von Raffael", die auf der Piazza Chigi "ein junger Mann mit farbigen Kreiden auf den Asphalt" malt, erinnert den Erzähler an jenes Bild, das "jahrzehntelang als Heiligenbild über unseren Köpfen im Kinderzimmer hing" und in weiterer Folge an sein allabendliches Gutenachtgebet, das "Schutzengelmein" (F, 152). Als er in Rom das "schnelle Aufspringen eines Mädchens nach dem Sturz mit ihrem Moped auf der Straße vor dem Markt" beobachtet, kommt ihm flugs ein eigener Fahrradunfall in den Sinn, in dessen Verlauf er als Kind "vom Drahtesel in den frisch gesäten Acker neben der Friedhofsmauer" gefallen war (F, 94f.), "am Markt herumflatternde[...] knisternde[...] Orangenpapiere" lassen ihn schließlich an jene "Blutorangenpapiere aus Sizilien" denken, die er als Kind in Kärnten gesammelt hatte, um sie "als Lesezeichen für [s]eine Karlmaybücher" zu verwenden ( $F, 93)$.

Eindrücke seines Romaufenthaltes ergeben zusammen mit Erinnerungen an die Kärntner Kindheit einen mehrschichtigen Erzählkosmos, in dem Ausgangspunkt und Ziel der Reise des Erzählers in eins verschmelzen.

Eine von Kindheitserlebnissen ausgehende thematische Fixierung bestimmt den Charakter der Reisen der Erzählerfiguren in den Büchern Josef Winklers mit, was zur Folge hat, dass die Reiseziele nicht in einem ethnografischen oder touristischen Sinn als solche kenntlich gemacht werden und gleichsam zur "Nebensache" ${ }^{14}$ werden. Ludwig Harig schreibt als Rezensent von Friedhof der bitteren Orangen, die "Suche nach den Spuren des Leidens" sei "diesmal eine italienische Spurensuche, bei der aber der Pfadfinder Winkler fortwährend in die Fußstapfen seiner Kärntner Dorfleute" tappe ${ }^{15}$ und Winklers Verlagslektor bei Suhrkamp, Hans-Ulrich Müller-Schwefe kommt es vor, als sei der Schauplatz von Domra, dem 1996 erschienenen Indien-Roman Winklers, Varanasi, "ein Vorort von Kamering", dem Geburtsort des Autors, "und doch viel mehr":

große, vielleicht glückliche Herausforderung durch eine andere Welt - die, im nachhinein, geradezu unheimlich gut zu Winklers Leben und Schreiben zu passen scheint. Vielleicht hat Winkler entdeckt, daß das Dorf seiner Kindheit - und eben nicht nur symbolisch, stellvertretend, sondern wirklich, ,in echt ${ }^{\star}$ - die ganze Welt ist; ungeheure Verengung dies; ungeheure Erweiterung ${ }^{16}$.

Primärer Grund für die Schauplatzwechsel im Werk Winklers ist die Aussicht, unbedrängt schreiben zu können, Zeit zu haben und eine schützende Entfernung zwischen sich und die Anfeindungen der Dorfbewohner zu legen. Vordergründig scheinen sich dadurch zunächst noch keine neuen Perspektiven aufzutun. Es fallen allerdings, und darin scheint Friedhof der bitteren Orangen tatsächlich eine Zäsur im Werk Josef Winklers zu markieren, zahlreiche Beschreibungen auf, die sich einer topografisch festlegbaren Rückführung nach Kärnten verweigern. Es handelt sich dabei offenbar um eine neue Qualität der Beobachtungen derselben Dinge und Vorgänge : Eine neuartige Fixierung auf die zentralen Themen wie Kirche und Tod führt letztlich zu einer manisch exakten, multiperspektivischen Erörterung, die immer mehr die Rolle der monomanischen Beschreibungen der Kindheit am Kärntner Bauernhof einnimmt. Die genannten Themenkreise beginnen sich in Friedhof der bitteren Orangen langsam zu eigenständigen Komplexen zu entwickeln. Salopp formuliert, erscheint es so, als reise der Erzähler seinen Themen nach, nachdem er sie in seinem bisherigen Lebenszusammenhang erschöpfend ausgebeutet hat. So verstanden, ist die Reise nach Italien, die eben auch in das "Zentrum der katholischen Kirche" führt, nicht nur eine Flucht aus der Kärntner Enge, sondern adäquate Folge inhaltlicher Interessen, ebenso 
wie jene nach Frankreich und Nordafrika, den Spuren Jean Genets folgend, oder, später, jene zahlreichen Aufenthalte im indischen Varanasi, wo der Erzähler den Totenkult akribisch festhält.

Wie Manfred Weinberg ausführlich dargelegt hat, stellt Friedhof der bitteren Orangen "eine präzisierbare Etappe" von Winklers "hin und weg von Kärnten" dar, eines Prozesses, der in der Novelle Natura Morta, in der Kärnten nicht mehr vorkomme, seinen vorläufigen Abschluss finde:

Mehr als je zuvor gibt sich dieser Roman [d.i. Friedhof der bitteren Orangen, Anm.d.V.] als Rede über die italienische Fremde. Die in ihm etwa beschriebenen Totenrituale von Bauern aus der Nähe Palermos sind Ausdruck eines ländlichen Katholizismus, der dem aus Kärnten bekannten zwar nahe steht; beschrieben wird hier aber eben nicht mehr das Eigene, sondern nur noch das ihm ähnelnde Fremde - ohne daß dabei noch ausdrücklich auf Kärnten zurückverwiesen würde. An die Stelle einer der Beunruhigung durchs Eigene geschuldeten - wörtlichen Ekstase tritt deshalb auch ein beruhigtes Abschildern [...]. [...] Fiel in den früheren Büchern Winklers die Ebene des Realen zugunsten einer ekstatischen Imagination/Vision aus, so hält er sich nun an das Beobachtete, beschreibt es aber hier auf eine Weise, die zeigt, daß die Realität auch nichts anderes ist als Imagination: jeder unscheinbare Augenblick ein potentiell ekstatischer ${ }^{17}$.

Mit dieser Veränderung wird umso mehr deutlich, dass Winklers Erzählweise der topographischen Fixierbarkeit realer Räume bestenfalls sekundäre Bedeutung zugesteht. Ihm geht es offensichtlich um etwas anderes, und das beeinflusst die Art, in der berichtet wird, fundamental und führt $\mathrm{zu}$ folgendem vorläufigen Schluss: Gleichgültig, ob Winklers Erzählerfiguren in Kärnten, Rom oder Indien sind, berichten sie auf eine ähnliche Weise von vergleichbaren Themen. Was sie reizt, sind Rituale, Kulte und Verhaltensweisen. Der Raum ist - wenn überhaupt - die Bühne, die die Anregungen liefert:

Gleichgültig, ob es sich um Kamering, Klagenfurt, Rom, Paris oder Benares handelt, die Empirie, die in Winklers Texten erscheint, hat zuerst und zuletzt eine metaphorische Substanz und sie befindet sich damit von vorneherein in Fundamentalopposition zu allen festgelegten Realitäten in Kamering, Rom oder sonstwo in der Welt ${ }^{18}$.

2

Großaufnahme. Breit ist der Lido von Venedig zu sehen. Ein Aquarium steht am Meeresstrand. Die Kamera fährt auf das Aquarium zu und fixiert die Fische. Durchs Glas des Aquariums sieht man die aufgepeitschten Meereswellen. Während die Kamera auf die Fische zufährt und einen Fisch in Großaufnahme fixiert, wird derselbe Fisch in der ersten Überschneidung auf dem Markt gezeigt, wie ihm der Fischer mit dem Messer den Bauch aufschlitzt. Anstatt den Fisch zu bezahlen, legt der Käufer Geld in die Handschale eines Bettlers, der an einer Straßenecke liegt. Mit einer weißen Maske aus der Commedia dell'Arte geht er im strömenden Regen auf den leeren Markusplatz. Ringsum hört und sieht er die Musikanten, die sich vor dem Regen schützend unter den Arkaden aufhalten. Eine Lampe pendelt in der Mitte des Platzes. Das schwankende Licht stößt immer wieder auf den Toreingang der Kathedrale und zurück. Katzen in den engen Gassen, Katzen auf den Brücken. In einer Halle läßt sich eine Frau porträtieren. Sie bemüht sich, den schönsten Ausdruck ihres Gesichts zu zeigen. Verwirrt geht er durch die Hallen, als suche er sein eigenes Kohlebild, für das er nie Portrait gestanden hat. Er geht durch die hellbeleuchteten Gassen, blickt in die Schaufenster und den verschiedenartigsten Puppen ins Gesicht. Er stößt unter den Arkaden des Markusplatzes mit einem 
Mädchen zusammen, entschuldigt sich nicht, denn er hat den Tod vor Augen. Auf dem Markusplatz steht er mit einer Dreibeinkamera, die mit einem schwarzen Tuch verhüllt ist. Der Fotograf wirft das Tuch über den Kopf, tritt hervor und zeigt ihm ein schwarz eingehülltes Kruzifix. Bildschnitt ${ }^{19}$.

Diese Textstelle aus einem frühen, in der Literaturzeitschrift manuskripte veröffentlichten Text gibt eine Richtung für spätere Italien-Blicke in Winklers Büchern vor. Typisch ist der Aufbau der Beobachtung: Ausgehend von einem Blick auf Aquariumsfische startet eine kleine Erzählung mit mehreren Schauplatzwechseln, Figuren und zahlreichen, surreal anmutenden Bildern. Der Beobachter folgt zuerst dem Fisch auf den Markt, dann dem "Käufer" desselben auf einer wirren Wanderung durch Venedig, vorbei an Masken, Bildern, Puppen und Menschen. Unweigerlich fühlt man sich an Thomas Manns Tod in Venedig erinnert und an Gustav Aschenbach im kranken, mit Todessymbolen überfrachteten Venedig.

Was auffällt, ist die Methodik der Verfremdung, mit der Winkler diese ohnehin bereits befremdende Beobachtung zusätzlich einrahmt: Zunächst ist festzuhalten, dass der Blick durch die Linse einer Kamera erfolgt, was die Unbefangenheit des Blicks grundsätzlich in Frage stellt, neue mediale Gesetzmäßigkeiten ins Spiel bringt und es letztlich sogar möglich erscheinen lässt, dass man es bei den beobachteten Figuren mit Schauspielern in gestellten Szenerien zu tun hat. Winkler bedient sich jedoch eines weiteren Kunstgriffs, der die Glaubwürdigkeit der beschriebenen Beobachtung zusätzlich vermindert: Der Blick ist von Beginn an im Wortsinn gebrochen, da er durch die Glaswände und das Wasser eines Aquariums erfolgt. Zudem erzeugt eine solche Art des Sehens Distanz - eine Technik, die Josef Winkler in seinen Büchern immer wieder verwendet ${ }^{20}$. Der Filmzusammenhang und die stark eingeschränkte Sicht, die letztlich surreale, bedrohliche Bilder freigibt, lassen einen Vergleich mit Federico Fellinis Film Roma zu, an dessen Beginn das Kamerateam in die Stadt fährt und die zunehmend surreal anmutende Welt außerhalb des Autos nur durch einen dichten, vom Regen hervorgerufenen Wasserschleier wahrnimmt.

Winkler stellt also auf mehrere Arten Distanz her, die den Beobachter und zugleich den Leser von seinen Objekten entfernt. Im Grunde genommen sind hier nicht einmal «Blicke » dargestellt - auch wenn uns der Text dies unentwegt glauben machen will -, das optisch Wahrnehmbare beschränkt sich auf ein Aquarium, die darin befindlichen Fische und, vordergründig als herkömmlicher Schauplatz vorgestellt, Venedig, dessen bloße Nennung die Herstellung verschiedenartiger inhaltlicher oder intertextueller Bezüge forciert. Während der Ort an sich angesichts des mehrfach verfremdeten, eher nach innen als nach außen gerichteten Blicks offenbar keine Bedeutung hat, erhalten die Ortsbezeichnungen eine wesentliche Funktion zugewiesen, indem sie es sind, die die Assoziationen des Lesers und Mitbeobachters lenken.

Bereits in diesem frühen Text Winklers wird somit deutlich, wie der Autor mit Schauplätzen umgeht : Sie sind nicht als Reiseziele zu verstehen, die von den Figuren der Texte aufgesucht werden, so wie reale Figuren reale Orte aufsuchen, sondern Kulminationspunkte von Assoziationslinien, Stellen, an denen Traditionen, Geschichten, Themenkreise, aber auch Klischees und Vorurteile zusammentreffen. Winklers Erzählerfiguren sind stets Getriebene, die an derartigen Assoziationspunkten hängen bleiben.

Die in Josef Winklers Texten dargestellten Vorgänge erheben keinerlei Anspruch auf Nachvollziehbarkeit und Glaubwürdigkeit. Thematisiert wird offenbar ein Bereich, der sich jenseits der allgemein sichtbaren Oberfläche befindet und sich nur der 
Erzählerfigur öffnet. Nur deren Befindlichkeit steuert das in höchstem Maße subjektive Geschehen, was passiert, findet folgerichtig in deren Kopf statt. Ähnlichkeiten und Verwechslungen mit realen Ereignissen, Orten oder Personen sind allerdings, wie die kurz angedeuteten Assoziationsketten im oben angeführten Beispiel zeigen, keineswegs zufällig, vielmehr spielt der Text mit dem Leseverhalten und den Versuchen, sich in den ausgelegten Irrwegen zurechtzufinden.

$\mathrm{Zu}$ diesem Spiel mit den Lesern gehört auch, dass Winkler die Erzählweise seiner Figuren in den Texten immer wieder thematisiert und zugleich hinterfragt und damit eine Art von Poetik mitliefert. Ein Beispiel dafür ist etwa ein programmatischer Satz aus dem Roman Muttersprache, in dem der Erzähler seinen außergewöhnlichen Blick als seine Art, nur das zu sehen, was andere nicht sehen wollen oder können, definiert: "Ich rede nur mehr über die anderen Dinge und lasse die Dinge, über die man redet, aus [...]" ${ }^{21}$. Die Perspektive, die diesen Blick ermöglicht, ist in einem anderen, sehr frühen Text beschrieben:

Verschiedene Bilder aus meiner bäuerlichen Vergangenheit formuliere ich in die Zeitform der Gegenwart hinein, da diese Erlebnisse noch heute in irgendeiner Form meines Handelns und Denkens spürbar sind. Das folgende Bild, das in der Vergangenheitsform steht, ist vielleicht eine Erinnerung oder Fantasie, die meine fortschreitende Sprache aufgeweckt hat. Oft vollzieht sich dieser Zeitenwechsel in einem einzigen Satz. Ich würde diese Art von Texten als « Reliefprosa » bezeichnen: der Wechsel der Zeit im einen und einzigen Erlebnis erinnert an übereinandergelegte Klarsichtfolien auf denen verschiedene Bergschichten eingezeichnet sind. Sieht man sie aus der Vogelperspektive, erkennt man die Strukturen dieses Berges ${ }^{22}$.

In dieser "Reliefprosa" ist der Erzähler nicht an physikalische Gesetze gebunden. Er steht - im wörtlichen Sinn - "darüber", was jedoch nicht heißt, dass er alles unter Kontrolle hat. Auch er blickt auf Ungeordnetes:

Der "böse Blick", Modus der Verräumlichung des Sturzes in Zeitschächte, macht aus den einzelnen Zeitstufen Trümmerlandschaften, Oberflächen, auf denen nebeneinander und durcheinander liegt, was durch den Fortlauf der Zeit überwunden schien ${ }^{23}$.

Als Folge dieser Perspektive wird der Beobachter selbst immer wieder überrascht, Dinge, Erinnerungen kommen zum Vorschein, die ihn überfordern, denn er will alles sehen und auch verarbeiten:

Links und rechts von mir flitzten alle möglichen Gesichter an mir vorbei, und ich dachte nur mehr daran, die Stazione Termini so schnell wie möglich zu verlassen, denn die Motive, die ich sah, überschlugen sich und purzelten mir davon, ich mußte verschwinden. (F, S. 140)

"Vogelperspektive" hat also bei Winkler nichts mit größerer Übersicht und in weiterer Folge Kontrolle zu tun, der Begriff veranschaulicht nur, wie eng die Zeitstufen in der Wahrnehmung des Erzählers beieinander liegen: Vergangenheit und Gegenwart vermischen sich, er "sieht" alles zugleich, ob er das will oder nicht ${ }^{24}$.

Für den Leser bedeutet die "Vogelperspektive" des Erzählers zusätzliche Arbeit: Winklers Texte erfordern genaue Konzentration und mehrmaliges Lesen.

In den Bildern tauchen Gehalte auf, deren Wertigkeit sich mitunter erst in Kenntnis später erzählter Sequenzen erschließt. Der Erzähler arbeitet, wenn er erinnert, schon mit einem Figurenerleben, das dem Leser noch gar nicht vermittelt wurde, ihm manchmal nie vermittelt wird. Er organisiert das Material sich und dem Leser nicht sinnvoll, sondern überfliegt es ${ }^{25}$. 
Anhand eines herausragenden Beispiels, des neapolitanischen "Camposanto delle Cedrangolette", soll im Folgenden gezeigt werden, wie Josef Winkler seine Schauplätze zunächst als reale Orte vorstellt, um sie in der Folge im Wortsinn in Literatur umzuwandeln, eine Vorgangsweise, die es von vorneherein unangemessen erscheinen lässt, überhaupt noch von "Schauplätzen" zu sprechen.

Italiens Katholizismus steht in Friedhof der bitteren Orangen in zwei, einerseits mit dem Tod, andererseits mit dem Medium Buch eng zusammenhängenden Ausformungen im Mittelpunkt: dem Titel gebenden Friedhof und dem Notizbuch mit einem Bild aus den Kapuzinerkatakomben in Palermo am Umschlag. Wie bereits im Klappentext zu lesen ist, bestattet Winkler die im Text beschriebenen Toten "Buchseite für Buchseite in den Grüften seines einem neapolitanischen Armenfriedhof, dem Campo Santo della Pietà, nachgebildeten Romans." ${ }^{26}$ Das Buch soll demnach eine veränderte, dislozierte Version des realen "Friedhof der bitteren Orangen" sein, es ist nach Der Ackermann aus Kärnten ${ }^{27}$ das zweite nach einem topologischen Konzept entstandene Buch des Autors ${ }^{28}$.

Der "Campo Santo delle Cedrangolette" in Neapel ${ }^{29}$, das Vorbild für Winklers "Buchfriedhof", wird im Roman immer wieder genannt und mehrmals beschrieben, am genauesten gleich bei der ersten Erwähnung :

War der Verstorbene arm, so wurde er in Neapel zum völlig ungeschmückten Campo Santo della Pietà gebracht, der sich aus 365 numerierten Gräbern zusammensetzte, in denen kalendarisch die Toten des Tages ohne Särge, vollkommen nackt, begraben wurden. Am ersten Jänner des darauffolgenden Jahres wurde die Grube, auf der die Steinplatte mit der Nummer $1 \mathrm{lag}$, wieder geöffnet, und die Neuverstorbenen wurden auf die verwesenden Leichen und Gerippe vom ersten Jänner des vergangenen Jahres geworfen. Nackte Kinder und Greise lagen übereinander. Statt ein paar Händen voll Erde wurde eine Schaufel voll Kalk auf die Toten geworfen. Die Gewänder, von denen die Toten auf dem Friedhof befreit wurden, verkauften Bettler in den Straßen Neapels. Es war, wie eine Chronik berichtete, ein Düngerhaufen, auf den die tägliche Ernte aus den Fondachi, den Spitälern und Gefängnissen geworfen wurde. Nachdem diese Bestattungsweise abgeschafft war, wurde ein Orangenhain über den Toten errichtet, und der Campo Santo della Pietà wurde in den Campo Santo delle Cedrangolette, den Friedhof der bitteren Orangen umgetauft. Heute wird dieser Friedhof Cimitero delle Fontanelle genannt. (F, S. 43)

Die Bestatteten sind demnach nackt, es gibt keine Särge, die Zersetzung der Körper wird durch die Beigabe von Kalk beschleunigt, ab dem Zeitpunkt der Beisetzung ist der Leichnam nur noch "Dünger"; In der Behandlung der einzelnen Toten gibt es keine Unterschiede, die Nummerierung der Gräber erfolgt "automatisch", Kleidung und alles, was den Toten als Lebenden gehört hat, bleibt unter Lebenden. Dies sind im Wesentlichen die Besonderheiten des Friedhofs ${ }^{30}$.

Es fällt auf, dass hier nur die Verfolgten, die Opfer bestattet werden. Der Erzähler, der sich selbst ausdrücklich zu den Außenseitern zählt ${ }^{31}$, solidarisiert sich mit ihnen, auch wenn er sich seinen eigenen Tod meist anders vorstellt: Einmal schreibt er zwar, er möchte "im Cimitero delle Fontanelle [also im Friedhof der bitteren Orangen] begraben werden und unter den Wurzeln eines Zitronenbaums liegen" (F, S. 181), an anderen Stellen will er nach dem Tod aber als Lesezeichen weiterleben, in einem Sarg, der aus den Buchdeckeln seiner Lieblingsbücher gemacht ist (F, S. 419-420), oder er sieht seinen Kopf als Mahnmal über den Dächern seines Heimatdorfes schweben (F, S. 408) ${ }^{32}$. 
Natürlich erfährt man in Winklers Buch nichts von den Menschen, die im realen Friedhof begraben liegen und ebenso wenig "wirklich" sind seine "Bestattungen". Wieder verschiebt sich die Perspektive weg vom namentlich genannten Ort hin zu einer metaphysischen oder genauer: literarischen Existenz. Winklers Erzähler erzeugt, indem er den Friedhof zu seinem "Buchfriedhof" macht, allerdings auch ein Gegenmodell zu allen bestehenden Friedhöfen, was, sozusagen als "realen Rest", einem gehörigen Maß an Kritik Platz lässt. Dahinter steckt das Konzept, den Opfern, den Unschuldigen, die weltliche Machthaber, vor allem die katholische Kirche, auf dem Gewissen hat, einen Zufluchtsort anzubieten. Passenderweise erhalten in seinem Friedhof daher jene Personen keinen Platz, die an der Unterdrückung anderer mitwirken oder diese billigen ${ }^{33}$.

Der Umgang des Erzählers mit den Toten erscheint, was die Technik der Bestattung anbelangt, durchaus konventionell - er folgt darin gängigen Ritualen. Beispielsweise werden beinahe alle im Roman beschriebenen Verstorbenen überführt. Damit bedient sich der Erzähler eines Mittels, das er in seiner Kärntner Kindheit kennen gelernt hat: "Oftmals hörte ich als Kind, dass die Bewohner meines Heimatdorfes, die außerhalb Kärntens starben, überführt und ihr Leichnam der Heimaterde übergeben wurde." (F, S. 412)

Die Rolle der Grabsteine schließlich nehmen im Buchfriedhof die Kurztexte auf den Seiten 21-79 und 351-409 ein, die noch einmal die Geschichte eines jeden Toten wiedergeben. Mehrmals werden sie ausdrücklich als "Grüfte" bezeichnet ${ }^{34}$. Die Verstorbenen erhalten mit den Texten also endgültig etwas, was ihnen im wirklichen "Friedhof der bitteren Orangen" nicht zusteht: ein eigenes Grab und eine Grabinschrift.

Formal erinnern die Kurztexte an mittelalterliche Epitaphien, wie eine Beschreibung aus der von Winkler als Quelle genannten ${ }^{35} G$ eschichte des Todes von Phillippe Ariès zeigt:

Bis zum 14. Jahrhundert setzt sich das allgemein verbreitete Epitaph also aus zwei Abschnitten zusammen: der eine, ältere, ist eine Identitätsangabe, die den Namen, die Stellung, das Todesdatum und zuweilen ein kurzes Lobeswort mitteilt. In der Mehrzahl der Fälle wird hier angehalten und weder das Alter noch das Geburtsdatum erwähnt. Der zweite, im 14. Jahrhundert verbreitete Abschnitt ist ein an Gott gerichtetes Gebet für die Seele des Verstorbenen [... ${ }^{36}$.

Auch Winklers Kurztexte bestehen in den meisten Fällen aus einem Erzählteil, in dem die Todesart des Verstorbenen beschrieben wird, und einem Gebet.

Was in einem früheren Buch Winklers angekündigt wurde - "Mit meinen Büchern will ich ihnen Grabsteine und Inschriften setzen" ${ }^{37}$-, wird in Friedhof der bitteren Orangen umgesetzt. Und damit ergibt sich wiederum eine Klammer, die Kärnten und Italien, die dortigen und die hiesigen Menschen, Schicksale und Riten verbindet und den thematischen Zusammenhang klar über die topographischen Gegebenheiten stellt. Folgerichtig werden nun auch die beiden jugendlichen Selbstmörder Jakob und Robert, Zentralfiguren und Schreibanlass nicht nur von Winklers erstem Buch Menschenkind in den Buchfriedhof überführt (F, S. 417).

Vom Gegenmodell, dem Totenkult der katholischen Kirche, unterscheidet sich Winklers Buchfriedhof in mehreren Punkten, zentral ist aber jener, dass von "seinen" Toten etwas zurückbleibt, dass er ihnen ihre Individualität in Form ihrer eigenen Geschichte überlässt. Die Kirche verfährt, wie im Buch immer wieder dargestellt ist, anders: Spätestens mit dem Tod fallen alle Menschen dem Machtbereich der Kirche zu. Wenn sie nicht, wie der verstorbene Papst Johannes XXIII, von Kardinälen einbalsamiert 
werden, nachdem ihnen die Eingeweide herausgerissen worden sind (F, S. 226), enden sie in geweihter Friedhoferde. Der Akt des Begrabens gerät zu einem grausamen Ritual des Einfangens:

Der Sarg, in dem das tote Mädchen lag, war mit Nelkenköpfen schon überfüllt, als ich sah, daß sich das Mädchen im Sarg mehrmals hin und her wälzte, aber gerade in diesem Augenblick wollte seine Mutter den Sarg für immer schließen. Ich schrie, Aber das Mädchen lebt noch, man darf es nicht begraben! Sie rissen mir den Sarg aus der Hand und hoben ihn in eine bereits ausgeschaufelte Grube, in der vier splitternackte junge Totengräber Erde schaufelten. (F, S. 280) ${ }^{38}$

Im Buchfriedhof ist durch das eindeutige Ende des Lebens (ohne Konservierung durch Einbalsamierung oder Särge, ohne "ideologische" Festlegung durch geweihte Erde, ohne am-Sterben-hindern-wollen durch Trauerkränze) ein neuer Anfang möglich. Auch für den Erzähler ist der Tod also ein Neubeginn - hier unterscheidet er sich grundsätzlich nicht von der Kirche; was die Besonderheit seines "Modells" ausmacht, ist, dass die Wiedergeburt, die im neapolitanischen Vorbild "real" (in Form von Orangen) erfolgt, nun, im Buch, auf eine andere, "surreale" Ebene verlegt wird: die Beschriebenen leben quasi weiter, ihr realer Tod ist die Voraussetzung dafür; beschrieben in den Epitaphien ${ }^{39}$ Winklers, erwachen sie zu neuem, "literarischem" Leben. Dieses "Leben nach dem Tod", das man durchaus analog zum katholischen sehen kann, macht sie unsterblich - ein Privileg, das in der Kirche nur die Würdenträger (eben die in deren Chroniken "Beschriebenen") erhalten. Winkler macht keinen Unterschied zwischen den Toten und bestattet auch Würdenträger - seine Epitaphien werden nicht nach "weltlichen" Kriterien verteilt.

Das Buch als Chronik derer, die von den anderen Chroniken vergessen wurden ${ }^{40}$, ist also, genauso wie das reale Vorbild, der "Campo Santo della Pietà", ein "Düngerhaufen", ein Sammelbecken für die Armen und Ausgestoßenen. Was dem Erzähler an diesem Vorbild nicht gefällt, kann er in der Realität nicht ändern ("Einzig und allein durch die Literatur kann ich Korrekturen anbringen" ${ }^{41}$ ), im Buch kann er alle Utopien verwirklichen: er lässt den auf seinem Buch-Friedhof begrabenen Toten ihre Individualität.

\section{4}

Winklers Spiel mit der Verfremdung und Umwandlung von Schauplätzen gewinnt seine besondere Qualität nicht zuletzt aus dem Zusammenprall mit den Erwartungshaltungen der Rezipienten. Die Vielzahl an mit Italien verknüpften Assoziationsmöglichkeiten, einerseits eine Grundlage für das Funktionieren der Winklerschen Erzähltechnik, birgt andererseits natürlich die Gefahr des fundamentalen Missverstehens. In Friedhof der bitteren Orangen ist eine ähnliche Situation auf pointierte Art wiedergegeben:

Gestern habe ich am Petersplatz überhaupt nichts gesehen, sagte ein Tiroler Bauer zu mir, denn dem großen Bildschirm, den das italienische Fernsehen aufgestellt hatte und auf dem man den Papst die Ostermesse zelebrieren sehen konnte, war ich zu nahe und vom leiblichen Papst war ich zu weit entfernt! (F, S. 124-125)

Die hier beschriebene Gefahr eines unscharf empfundenen Blicks, der doch "vor Ort" und "aus nächster Nähe", also vermeintlich unbestechlich und exakt ausfallen sollte, ist im Fall Josef Winklers in besonderer Weise gegeben. Selbst professionelle Leser haben angesichts der Lektüre des "anderen" Italien-Buches Friedhof der bitteren Orangen 
geradezu verstört reagiert und den Autor persönlich verunglimpft ${ }^{42}$. Dass die Blicke auf Rom, die Winkler liefert, nicht viel mit den bekannten und erwünschten der meisten Italien-Reisenden zu tun haben (und auch wenig mit der so genannten "Ethnopoesie" ${ }^{43}$ ), ist wohl klar. Im Verlauf seiner Bücher ist, wie anfangs erwähnt, aber auch in dieser Hinsicht eine interessante Entwicklung auszumachen: War bereits Friedhof der bitteren Orangen ein Buch, in dem mehr von Italien zu sehen war als in den ganz frühen Texten Winklers, ist der Erzähler, wie Weinberg festhält, in den zuletzt erschienenen Büchern wirklich 'in einem 'fremden' Land angekommen und beschreibt es tatsächlich ethnographisch genau". Gleich geblieben ist dabei sein Interesse, das nach wie vor nicht der "Fremde und [den] Fremden]" gilt, sondern dem "(]Verhältnis von) Leben und Tod" ${ }^{44}$. Vor allem im Hinblick auf die 2001 erschienene und wieder in Rom handelnde Novelle Natura Morta ${ }^{45}$ wurde darauf verwiesen, dass Winklers "metaphysische" Art der Wahrnehmung realer Räume sich hin zu einer neuen, auch für Ethnologen fruchtbaren Darstellung entwickle:

Die bloß "metaphorische" Realität ist nun keine die Wirklichkeit ersetzende mehr; doch ethnographisch im Sinne eines genuinen Forschungsinteresses ist sie nicht. [...] Das detailliert Beschriebene öffnet sich auf etwas ihm Zugrundeliegendes hin, das in ethnographischen Berichten gemeinhin nicht in den Blick gerät. Solche "Öffnung" gelingt nur der Literatur ${ }^{46}$.

\section{NOTES}

1. Hans-Ulrich Müller-Schwefe: "Randschriftlich. Zu Josef Winklers Domra", in: beigesellt. fernwesend. Beiträge zu Josef Winkler und seinem Werk, hrsg. von Reinhard Kacianka, Klagenfurt, Kitab, 2004, S. 56.

2. Josef Winkler: Friedhof der bitteren Orangen, Frankfurt am Main, Suhrkamp (suhrkamp taschenbuch 2171), 1993. Weitere Zitate aus dem genannten Buch sind im Lauftext in runden Klammern wiedergegeben und mit dem Kürzel F gekennzeichnet.

3. Auch Andrea Kunne hat darauf verwiesen. Andrea Kunne: Heimat im Roman: Last oder Lust? Transformationen eines Genres in der österreichischen Nachkriegsliteratur, Amsterdam/ Atlanta, GA., Rodopi (Amsterdamer Publikationen zur Sprache und Literatur, Band 95), 1991, S. 257.

4. Vgl. Martin Lüdke: "Kärntner Jagdszenen. Josef Winklers Roman Friedhof der bitteren Orangen", Die Zeit, 05. 10. 1990, S. 6: "Er schreibt, seit er schreibt, seit gut zehn Jahren, an einem Buch."; Gerhard Moser: "Bittere Orangen über 365 Gräbern. Josef Winkler arbeitet an einem neuen Roman", Wiener Zeitung, 15.09.1989, Beilage, S. 8: "Bereits mehr als tausend Seiten - oder, in kleineren Einheiten gerechnet, genau vier Romane - umfaßt seine ichbezogene, metaphernreiche Auseinandersetzung mit dem Oberkärntner Heimatdorf, mit dem Bauernhof und der Vaterfigur". Weiters: Klara Obermüller: "Die zwei am Kälberstrick. Josef Winkler schreibt weiter an dem Roman seines Lebens", Frankfurter Allgemeine Zeitung, 18.07.1987, S. 5: "Josef Winkler schreibt an ein und demselben Buch, ein Leben lang vermutlich."

5. Joachim Campe, zit. nach Agnes Hüfner: "Auf Vernichtung aus. Josef Winkler kehrt in die Trübnis seiner Vergangenheit zurück”, Süddeutsche Zeitung, 29.07.1987. 
6. Karl Nitsch: "Im Bann des Tabus. Notizen zur Alptraumprosa Josef Winklers", Wiener Blätter 8/90, S. 23.

7. Dirck Linck: Halbweib und Maskenbildner: Subjektivität und schwule Erfahrung im Werk Josef Winklers,Berlin, Verlag rosa Winkel (Homosexualität und Literatur; Bd. 7), 1993, S. 212; ähnlich auch Gabriel Grüner: "Bilder einer großen Verstörung. Zwei junge Kärntner in der Tradition Thomas Bernhards - Josef Winkler und Alois Hotschnig”, Deutsches Allgemeines Sonntagsblatt, 08.02.1991.

8. Ernst Grotoholsky: "Wo einem ein Kopf in den Satz schießt oder: Wieder dasselbe Thema aber wieder ganz anders" [Ein Gespräch mit Josef Winkler], in: Josef Winkler, hrsg. von Günther A. Höfler und Gerhard Melzer, Graz/ Wien, Droschl (Dossier 13), 1998, S. 19-20.

9. Vgl. dazu vor allem: Franz Haas: "Ketzergebete oder: Josef Winklers poetologische Herbergssuche", in: Josef Winkler, a.a.O., S. 39-54.

10. Josef Winkler: Der Leibeigene,Frankfurt am Main, Suhrkamp (suhrkamp taschenbuch 1731), 1990, S. 253.

11. So nannte der Suhrkamp Verlag die als Trilogie zusammengefassten ersten drei Romane Winklers, Menschenkind, Der Ackermann aus Kärnten und Muttersprache.

12. Vgl. dazu Alois Brandstetter: "Enzn Sepp alias Josef Winkler", in: beigesellt. fernwesend. Beiträge zu Josef Winkler und seinem Werk, a.a.O., S. 25-26.

13. Ernst Grotoholsky: "Wo einem ein Kopf in den Satz schießt oder: Wieder dasselbe Thema aber wieder ganz anders", a.a.O., S. 20.

14. Franz Haas: "Ketzergebete oder: Josef Winklers poetologische Herbergssuche", in: Josef Winkler, a.a.O., S. 51.

15. Ludwig Harig: "Ein Nagelbrett statt Kerzen. Josef Winklers Roman Friedhof der bitteren Orangen". Zitiert nach: Josef Winkler, a.a.O., S. 146 (Erstdruck in: Die Frankfurter Rundschau, 04.10.1990).

16. Hans-Ulrich Müller-Schwefe: "Randschriftlich. Zu Josef Winklers Domra", in: beigesellt. fernwesend. Beiträge zu Josef Winkler und seinem Werk, a.a.O., S. 46.

17. Manfred Weinberg: "Hin und weg von Kärnten. Josef Winkler und die Fremde", in: Ethno/ Graphie. Reiseformen des Wissens, hrsg. von Peter Braun und Manfred Weinberg, Tübingen, Gunter Narr Verlag, 2002, S. 340-342.

18. Klaus Amann: “Josef Winkler. Allerheiligenhistoriker, Karfreitagspsychologe, Christihimmelfahrtsphilosoph, Mariaempfängnisneurotiker. Eine biographisch-dokumentarische Skizze", in: Josef Winkler, a.a.O., S. 209.

19. Josef Winkler: “Die Harry's Bar in Venedig und Alfred Kolleritsch", manuskripte 69/70, 1980, S. 22.

20. Vgl. etwa Hans-Ulrich Müller-Schwefe: "Randschriftlich. Zu Josef Winklers Domra", a.a.O., S. 48.

21. Josef Winkler:Muttersprache, Frankfurt am Main, Suhrkamp (suhrkamp taschenbuch 1044), 1984, S. 34.

22. Josef Winkler: “Nachwort zu 'Blitzlichter aus dem Dorfe K.”, manuskripte 62, 1978, S. 78.

23. Dirck Linck: Halbweib und Maskenbildner: Subjektivität und schwule Erfahrung im Werk Josef Winklers, a.a.O., S. 87.

24. Dirck Linck: Halbweib und Maskenbildner: Subjektivität und schwule Erfahrung im Werk Josef Winklers, a.a.O., S. 89.

25. Antonio Fian: "Hölle, verlorenes Paradies. Zu Josef Winklers Kärnten-Romanen", manuskripte 124, 1994, S. 93.

26. Ludwig Harig, in: Josef Winkler: Friedhof der bitteren Orangen, a.a.O., S. 2.

27. Josef Winkler: Der Ackermann aus Kärnten, Frankfurt am Main, Suhrkamp, 1984.

28. Vgl. Klaus Kastberger: “Lebenslängliches Sterben”, Falter 10, 1991, S. 10. 
29. Franz Haas: "Auch er war in Arkadien", in: beigesellt. fernwesend. Beiträge zu Josef Winkler und seinem Werk, a.a.O., S. 62.

30. Mit den Toten im Friedhof der bitteren Orangen, von denen nichts zurück bleibt, stellt Winkler ein Gegenbild zu den leitmotivisch immer wieder erwähnten Kapuzinerkatakomben in Palermo dar, in denen die Leichen ihre schönste Kleidung tragen und, bestückt mit religiösen Symbolen, durch die Abgeschlossenheit des Kellers nur sehr langsam verwesen. Jahrzehnte lang bieten sie so ein makaberes - und touristisch bestens verwertbares - Bild.

31. Vgl.: "Unter den Außenseitern und Ausgestoßenen fühle ich mich wohl, aber ich weiß, daß ich es unter diesen Menschen auch deswegen aushalte, weil ich noch nicht ganz so heruntergekommen bin wie sie [...]. Wäre ich so vollkommen mittellos wie diese Menschen, würde ich versuchen, ihre Plätze zu meiden. Ich nütze meinen Vorteil aus. " Aus: Josef Winkler: Der Leibeigene, a.a.O., S. 300-301.

32. Weitere Stellen, in denen er über seinen Tod schreibt: F, S. 85, F, S. 180-182.

33. Ein Beispiel dafür ist die Hauptfigur eines früheren Buchs Josef Winklers, Die Verschleppung, Warwara Wassiljewna, die den Erzähler verbal angreift und sich damit auf die Seite der "Mächtigen" begibt. Der Erzähler erstattet ihr daraufhin alle ihre Geschichten zurück (F, S. 387-396).

34. "Gruft 24" (F, S. 412; dabei handelt es sich um den Kurztext auf Seite 53); "20. Gruft" (F, S. 414; Kurztext S. 45); “42. Gruft” (F, S. 415; Kurztext S. 78-79).

35. F, S. 182. Auch wenn der Autor in Winklers Buch nicht ausdrücklich genannt wird, ist davon auszugehen, dass es sich um dieses Buch handelt.

36. Philippe Ariès: Geschichte des Todes, München, Deutscher Taschenbuch Verlag, 1987, S. 280.

37. Josef Winkler:Muttersprache, a.a.O., S. 144.

38. Vgl. auch folgende Textstelle: "Der Tote lag in violetter Seide. Seine spitze Nase und seine Fingernägel hatten bereits einen bläulichen Farbton. Seine Wangen waren eingefallen wie die leichte Vertiefung eines goldenen Tellers, auf den der Priester den Leib Christi legt, die Hostie, auf der die Fingerabdrücke des regierenden Papstes eingeprägt sind, die er dem Sterbenden während seines Todeskampfes zwischen seine bläulichen Lippen schiebt. Der Leichenbestatter versiegelte mit einem Schweißapparat den Zinnsarg, der wie eine russische Puppe in einen Holzsarg gestellt wurde. [...] Wenn der Tote noch die Kraft hätte, seine zum Gebet geschlossenen Hände - wobei man nicht einmal weiß, ob er überhaupt wollte, daß seine Hände zum letzten Gebet geschlossen werden - zu öffnen, und den, der ihn beklagt, zur Strafe, weil er sich selber und niemand anderen beweint, mit in den Sarg zerren könnte, bevor er vom venezianischen Leichenbestatter zugelötet wird ! Aber er liegt vollkommen kraftlos im Sarg und ist nicht einmal fähig, die Fliegen zu verjagen, die sich an ihn heranmachen.” (F, S. 227-228).

39. Auch die Epitaphien des 14. Jhdts. entstanden aus dem "Bedürfnis, die eigene Identität im Tode zu bekräftigen" (Philippe Ariès: Geschichte des Todes, a.a.O., S. 278) - eine weitere Analogie zwischen den Kurztexten des Romans und ihren mittelalterlichen "Vorbildern".

40. Vgl. Manfred Müller: "Kein Wunder, daß ich zuerst das Böse sehe. Anmerkungen zur Wahrnehmung der Erzählerfiguren Josef Winklers", Was 84, Februar 1996, S. 49-50.

41. Josef Winkler: “Nachwort zu 'Blitzlichter aus dem Dorfe K.' ", manuskripte 62, 1978, S. 78.

42. Vgl. etwa Harald Hartung: "Kärnten ist überall. Josef Winklers Friedhof der bitteren Orangen", Frankfurter Allgemeine Zeitung, 11.12.1990 oder auch: Sigrid Löffler: "Josef Winkler: Friedhof der bitteren Orangen". Skript der ORF-Radiosendung "Ex Libris”, Oktober 1990 (Typoskript in der Dokumentationsstelle für Neuere Österreichische Literatur, Wien).

43. Narjes Khodaee Kalatehbali hat in einer interessanten Arbeit u. a. auf die ethnologischen Aspekte von Winklers Roman Domra. Am Ufer des Ganges hingewiesen. In: Narjes Khodaee Kalatehbali: Das Fremde in der Literatur. Postkoloniale Fremdheitskonstruktionen in Werken von Elias Canetti, Günter Grass und Josef Winkler, Münster, LIT Verlag, 2005, S. $168 \mathrm{ff}$.

44. Manfred Weinberg: "Hin und weg von Kärnten. Josef Winkler und die Fremde", a.a.O., S. 353. 
45. Josef Winkler: Natura Morta, Frankfurt am Main, Suhrkamp, 2001.

46. Manfred Weinberg: "Hin und weg von Kärnten. Josef Winkler und die Fremde", a.a.O., S. 353.

\section{RÉSUMÉS}

Die Erzählerfiguren in den Texten Josef Winklers reisen nicht als Touristen oder Ethnologen. Sie folgen Themen verschiedenster Art, die sie in früheren Büchern des Autors im heimatlichen Kärnten kennen gelernt haben. Die bereisten Orte erhalten ihre Bedeutung damit in einem metaphysischen Sinn, als Kulminationspunkte von Assoziationslinien, an denen Traditionen, Geschichten, Klischees und Vorurteile zusammentreffen.

Der 1990 erschienene Roman Friedhof der bitteren Orangen spielt, der Religions- und Todesthematik von Winklers Frühwerk folgend, in Italien. Am Beispiel des neapolitanischen "Camposanto delle Cedrangolette“ lassen sich die Mechanismen erkennen, mittels derer der Erzähler den realen Ort seinem Erzählkosmos einverleibt: In früheren Büchern erwähnte Verstorbene werden darin ebenso wie in kirchlichen Chroniken oder Geschichtsbüchern aufgefundene, von der Kirche oder anderen, weltlichen Instanzen geschändete Tote metaphorisch bestattet - aus dem Friedhof wird ein Buchfriedhof, aus dem realen Ort ein literarisches Denkmal.

Mit seinem Idealfriedhof definiert sich der Erzähler einerseits als machtvolle, korrigierende Instanz (wenngleich seine Macht "nur" eine literarische ist), andererseits vermag er auf diese Weise zugleich die Personen und Geschichten, die ihn im Frühwerk manisch verfolgt und zum Schreiben gezwungen hatten, zu bestatten und damit erstmals gleichsam abzuhaken.

Dans les textes de Josef Winkler, les narrateurs ne voyagent pas en touristes ou en ethnologues. Ils sont sur les traces de thèmes très divers, qu'ils ont rencontrés dans de précédents ouvrages de l'auteur dans leur Carinthie natale. Le lieu visité acquiert par là un sens métaphysique : point nodal de chaînes associatives, il est confluence de traditions, d'histoires, de clichés et de préjugés.

Le roman Cimetière des oranges amères, publié en 1990, se déroule en Italie : le choix de ce cadre découle de la prégnance de la religion et de la mort dans les œuvres de jeunesse de Winkler. L'exemple du cimetière napolitain du Camposanto delle Cedrangolette permet de percevoir selon quels mécanismes le narrateur incorpore le lieu réel à son univers narratif: des défunts mentionnés dans des œuvres antérieures de Winkler ainsi que des morts victimes du pouvoir religieux ou laïc, découverts dans des chroniques religieuses ou des livres d'histoire, y reçoivent une sépulture métaphorique - le cimetière se fait cimetière de papier, le lieu réel monument littéraire à la mémoire des disparus.

Ce cimetière idéal permet au narrateur d'une part de se définir comme une instance dotée de pouvoir, une instance corrective (même si son pouvoir n'est "que" littéraire), d'autre part d'enterrer les personnages et les histoires qui, dans les œuvres de jeunesse, l'ont poursuivi de manière obsessionnelle et contraint à écrire - et ainsi, pour la première fois, en quelque sorte, de tirer un trait.

In Josef Winkler's texts, narrators do not travel as tourists or ethnologists. They follow the traces of very various themes, that they met in the author's previous works in their native Carinthia. The visited place acquires thus a metaphysical sense: nodal point of associative chains, it is the 
convergence of traditions, histories, clichés and prejudices. The novel Graveyard of Bitter Orange, published in 1990 takes place in Italy: the choice of this place follows from the pregnancy of religion and death in Winkler's early works. The example of the Neapolitan graveyard Camposanto delle Cedrangolette enables to understand through which mechanisms the narrator incorporates the real place to his narrative universe: departed mentioned in Winkler's previous works as well as departed, victims of the religious or laic power, discovered in religious chronicles and history books, receive a metaphorical grave - the cemetery becomes paper cemetery, the real place becomes a literary monument in memory of the departed. This ideal cemetery enables the narrator on one hand to define himself as an authority holding the power and correcting (even if its power is "only" literary); on the one other hand to bury characters and histories that, in early works, chased him in an obsessional way and forced him to write and thus for the first time, so to speak, to turn over a new leaf.

INDEX

Mots-clés : voyage intérieur, Italie, religion, mort

oeuvrecitee Friedhof der bitteren Orangen, Cimetière des oranges amères

\section{AUTEURS}

\section{MANFRED MÜLLER}

Universität Wien 